\title{
Study of consumer fireworks post-blast residues by ATR-FTIR
}

Carlos Martín-Alberca ${ }^{a, b}$, Félix Zapata ${ }^{\text {a,b }}$, Héctor Carrascosa a ${ }^{\text {a }}$ Fernando E. OrtegaOjeda $^{\mathrm{b}}$, Carmen García-Ruiz ${ }^{\mathrm{a}, \mathrm{b}, *}$

a Department of Analytical Chemistry, Physical Chemistry and Chemical Engineering, Multipurpose Building of Chemistry, University of Alcalá, Ctra. Madrid-Barcelona Km. 33.6, 28871 Alcalá de Henares (Madrid), Spain.

${ }^{\mathrm{b}}$ University Institute of Research in Police Sciences (IUICP), University of Alcalá, Ctra. Madrid-Barcelona Km. 33.6, 28871 Alcalá de Henares (Madrid), Spain.

E-mail: carmen.gruiz@uah.es; carlos.martina@uah.es; felix.zapata@uah.es

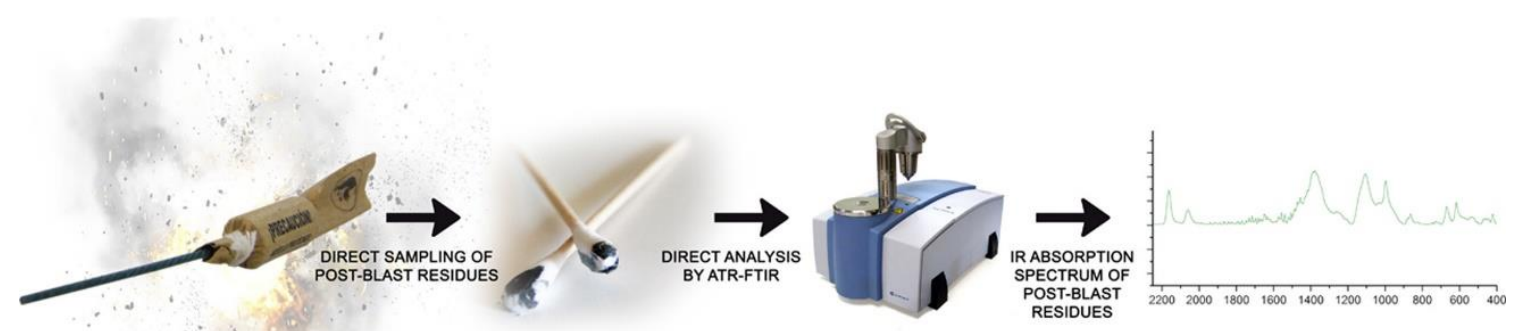

Cite: C. Martín-Alberca, F. Zapata, H. Carrascosa, F. E. Ortega-Ojeda, C. GarcíaRuiz, Study of consumer fireworks post-blast residues by ATR-FTIR, Talanta 149 (2016) 257-265. DOI: 10.1016/j.talanta.2015.11.070 


\begin{abstract}
Specific analytical procedures are requested for the forensic analysis of pre- and postblast consumer firework samples, which present significant challenges. Up to date, vibrational spectroscopic techniques such as Fourier transform infrared spectroscopy (FTIR) have not been tested for the analysis of post-blast residues in spite of their interesting strengths for the forensic field. Therefore, this work proposes a simple and fast procedure for the sampling and analysis of consumer firework post-blast residues by a portable FTIR instrument with an Attenuated Total Reflection (ATR) accessory. In addition, the post-blast residues spectra of several consumer fireworks were studied in order to achieve the identification of their original chemical compositions. Hence, this work analysed 22 standard reagents usually employed to make consumer fireworks, or because they are related to their combustion products. Then, 5 different consumer fireworks were exploded, and their residues were sampled with dry cotton swabs and directly analysed by ATR-FTIR. In addition, their pre-blast fuses and charges were also analysed in order to stablish a proper comparison. As a result, the identification of the original chemical compositions of the post-blast samples was obtained. Some of the compounds found were potassium chlorate, barium nitrate, potassium nitrate, potassium perchlorate or charcoal. An additional study involving chemometric tools found that the results might greatly depend on the swab head type used for the sampling, and its sampling efficiency. The proposed procedure could be used as a complementary technique for the analysis of consumer fireworks post-blast residues.
\end{abstract}

Keywords: ATR; Chemometrics; Fireworks; Forensic analysis; FTIR; Pyrotechnics; Post-blast residues; Sampling. 


\section{Introduction}

Consumer fireworks are small display pyrotechnic devices available on sale to the general public. They are designed to be lit up and produce some effects such as smoke, noise, colour, sparks or flames by combustion [1]. Some examples are firecrackers, bottle rockets or sparklers. They are made with clever chemical compositions and configurations, which are responsible for the pyrotechnic effects. The most frequently used chemical compounds are oxidizer agents such as nitrate, perchlorate or chlorate salts, fuels such as charcoal, magnesium or aluminium, and auxiliary substances such as potassium benzoate or strontium carbonate $[1,2]$.

There is high forensic interest in the determination of the chemical composition of consumer fireworks, from both intact items and their post-blast residues. This is important during investigations of caseworks related to vandalism acts, injuries, occupational accidents, illegal trade or improvised explosive devices made with their components [1, 3]. Although the analysis of this type of samples is challenging, forensic experts try to obtain a complete evidence characterization and/or identification by means of applying multiple specific analytical techniques based on different principles and methodologies [4]. Some complications for studying consumer fireworks include the fact that they may have complex configurations and compositions, like several fuses and charges, or that the total amount of pyrotechnic charge is usually low (0.1-2 g [5]), which means that the available amount of post-blast residues is assumed quite lower. Furthermore, studying post-blast residues is a daunting task because some unburnt material from fuses and charges, and reaction products from the original composition are normally mixed with inert materials such as clay plugs or cardboard [6].

Consequently, several specific analytical approaches for the analysis of consumer firework samples were proposed. Colorimetric methods were applied for the quantitative determination of given substances such as sulphur and chlorate in intact items [7]. Scanning electron microscopy/energy dispersive X-ray spectroscopy (SEM-EDX) was used to identify chemical elements in pre- and post-blast consumer firework samples, and to differentiate between pyrotechnic particles and gunshot residues [8-11]. Besides, several sensitive methodologies were proposed for the analysis of consumer firework samples by Capillary Electrophoresis (CE) with UV-vis detection [5, 12-14], and by a portable dual-channel CE with contactless conductivity detection [15]. These 
methodologies allowed the identification of their ionic composition. Moreover, it was even possible to associate the identified post-blast ions with their original reagents through the decomposition or combustion derived reaction products $[5,15]$. In recent years, the use of spectroscopic techniques has increased in this field especially because they can provide useful atomic and molecular information. Inductively Coupled Plasma, Atomic Fluorescence Spectroscopy and Atomic Emission Spectroscopy were applied to the identification of metallic elements in intact consumer fireworks [6, 16]. Mass spectrometry (MS) coupled to desorption-electrospray-ionization [17] and to laserelectrospray [18] were applied to the analysis of intact consumer fireworks and their postblast residues, respectively. Furthermore, it is well known that Raman and Fourier transform infrared (FTIR) spectroscopy are suitable techniques to analyse organic or inorganic explosives [19]. In fact, Raman spectroscopy and FTIR spectroscopy (used in transmittance mode) were also applied to detect the molecular composition of intact consumer fireworks [20]. Both techniques have very interesting characteristics in the forensic context, for instance, they allow fast result acquisition without sample destruction, and they are available as portable systems. However, to our knowledge, the use of these techniques for the analysis of post-blast residues of consumer fireworks has not been reported up to date. The reasons might include the challenging issue of analysing this type of samples and the lower sensitivity of these techniques compared with the other previously mentioned. Nevertheless, because they offer interesting strengths for the forensic field, these spectroscopic techniques should be further tested for the specific analysis of post-blast consumer fireworks. A crucial step to achieve the successful analysis of these samples by these techniques is the sampling step. In fact, up to date, several studies have involved the recovery of the explosives' organic and inorganic postblast residues from numerous surfaces using several swab types [21-23]. All these studies were devised for collecting and then extracting the analytes using solvents for their further analysis by separation techniques. However, they did not tackle the direct analysis of the residues remnant on the swab heads, which might implicate additional challenges.

The aims of this work were to obtain the spectra of several post-blast consumer fireworks samples using a simple and fast sampling and analysis procedure by a portable FTIR instrument with Attenuated Total Reflection (ATR) accessory, and to achieve the identification of their original chemical compositions. In order to accomplish these goals, the standard reagents of the main compounds found in consumer fireworks and their main 
combustion products were analysed. Afterwards, some items were exploded, their postblast residues were swabbed and immediately analysed by ATR-FTIR. In addition, preblast fuse and charge samples were analysed, and their spectra compared to their corresponding post-blast counter parts.

\section{Material and methods}

Standards, samples, materials and instrumentation

All standards were of analytical grade. Sodium nitrate, strontium nitrate, barium nitrate, potassium chlorate, sodium chlorate, potassium perchlorate, potassium thiocyanate, sodium thiocyanate, potassium cyanate, sodium cyanate, potassium sulphate, sodium sulphate, sodium thiosulphate, potassium thiosulphate, strontium carbonate and aluminium were obtained from Sigma-Aldrich (St. Louis, MO, USA). Potassium nitrate, Sulphur and copper oxide (II) were purchased from Labkem (Mataró, Barcelona, Spain). Wood charcoal (powder), sodium nitrite, calcium chloride and calcium carbonate were bought from Fisher Scientific (Loughborough, Leicestershire, UK).

Five different consumer fireworks were selected as samples. They were purchased in a local store (Alcalá de Henares, Madrid, Spain). The three simple firecrackers were named as F1, F2 and F3 (commercial names: 100petardos, cheroki junior and superfallero, respectively). One complex firecracker was named as F4 (commercial name: cracks). Finally, a bottle-rocket was named as R1 (commercial name: voladores surtidos). Table 1 collects the information provided by manufacturers about the used firework's composition. Additional chemical information of these samples was obtained in previous studies [5, 15].

Table 1. Composition of the consumer fireworks declared by manufacturers.

\begin{tabular}{cl}
\hline Consumer firework & Declared composition (charges) \\
\hline F1 & $\mathrm{Al}$; Sulphur; $\mathrm{KClO}_{4}$ \\
F2 & Sulphur; Magnalium; $\mathrm{Al} ; \mathrm{KClO}_{4}$ \\
F3 & $\mathrm{Al}$; Sulphur; $\mathrm{KClO}_{4}$ \\
& Cracking effect: $\mathrm{KClO}_{4} ; \mathrm{KNO}_{3} ;$ Sulphur; Charcoal; Magnalium; $\mathrm{CuO}$ \\
F4 & Green smoke: Sulphur; $\mathrm{Ba}\left(\mathrm{NO}_{3}\right)_{2} ; \mathrm{KClO}_{4}$ \\
& Thunder effect: $\mathrm{Al} ; \mathrm{Sulphur} ; \mathrm{KClO}_{4}$ \\
& Lift mixture: Sulphur; Charcoal; $\mathrm{KNO}_{3}$ \\
& Burst mixture: $\mathrm{Sulphur} ; \mathrm{Charcoal} ; \mathrm{KNO}_{3}$ \\
F5 & Green effect: $\mathrm{C}_{48} \mathrm{H}_{42} \mathrm{O}_{7} ;\left(\mathrm{C}_{2} \mathrm{HCl}\right)_{n} ; \mathrm{KClO}_{4} ;$ Magnalium; $\left.\mathrm{Ba} \mathrm{NO}_{3}\right)_{2}$ \\
\hline
\end{tabular}


Cotton swabs from three different manufacturers were tested in order to assess the effectivity of the swabbing system and the background signals produced by the swab head material. Specifically, they were obtained from Prionics AG (Schlieren, Zurich, Switzerland), Remel-Thermo Fisher scientific (Santa Fe Drive, P. O., USA), and BaikimRuher Iberica S.A. (Barcelona, Spain). It is important to note that the swabs from Prionics $\mathrm{AG}$ (named as $\mathrm{Cb}$ ) were designed for forensic evidence collection, whereas the swabs from Remel (named as $\mathrm{Sw}$ ) were designed for microbiology purposes, and the swabs from Baikim (named as Hy) were designed for personal care.

A compact portable ALPHA FTIR spectrometer with ATR sampling accessory (Bruker Optics Inc., Ettlingen, Germany) was used to analyse the samples.

\section{Sample preparation, sampling and analysis}

In order to obtain post-blast samples, two items of each consumer firework type were exploded on a steel plate (an open metallic sheet, named as Ms). On additional experiments, a glass surface (a large glass desiccator, named as Des) was considered. The methodology used to clean the steel plate and the glass surface between explosions was the reported in reference [5]. Briefly, the post-blast residues were obtained swabbing the surface with a dry cotton swab, trying to collect residues from all over the surface. Then, the dry cotton swab was placed and pressed directly on the cleaned diamond crystal surface of the ATR sampling accessory using the devices' mechanical press. Each swab was analysed thrice by changing (rotating) its position under the press, thus assuring a fresh measurement each time. The instrument was cleaned between analyses with a clean paper tissue and then with isopropanol-soaked tissues. Furthermore, the pre-blast samples of three additional items of each consumer firework type were also analysed. The following procedure was used for studying the non-exploded items: First, the fuse was separated from the main part of the item. The most external part of the fuse (not contaminated by the main charge of the item) was divided in two. One-half was selected for extracting its containing fuse-powder. Then, the extracted powder was analysed directly on the cleaned diamond crystal surface of the ATR sampling accessory. Afterwards, the item's main part (body) was opened carefully using a scalpel and its powdered content was emptied on a filter paper. Only a small amount (around $50 \mathrm{mg}$ ) of such powder was analysed. No other pre-treatment was performed on the samples. Some consumer fireworks contained more than one charge, thus each type of charge was 
separated for their further analysis, except for those cases where cross-contamination was observed with the naked eye. In those cases, they were mixed further and analysed as a mixed sample.

\section{Data acquisition}

IR absorption spectra were recorded from 4000 to $400 \mathrm{~cm}^{-1}$ with a spectral resolution of 4 (data spacing of $2.04 \mathrm{~cm}^{-1}$ ). For every measurement, the system performed 48 scans, which then were automatically averaged using the Opus 7.2 software (Bruker Optics, Ettlingen, Germany). In order to focus on the spectral fingerprint region, the spectral region selected for the data treatment was $2200-400 \mathrm{~cm}^{-1}$. In fact, all the characteristic bands for the inorganic oxidizers and the other components common in these samples are included within this range. Afterwards, a rubber band baseline correction ( $n=64$ points and 15 iterations), and a range normalization between 0-2 (intensity value) were performed. The data was subsequently imported and analysed in the SIMCA 14 software (Umeå, Sweden). For this purpose, different chemometric tools like principal component analysis (PCA) were used. The PCAs were performed to visualize in a straightforward fashion how similar or different the spectra were, and to obtain a first overview of the main sources of variability. First, the noise in the spectra was reduced using the SavitzkyGolay built-in function ( $2^{\text {nd }}$ order polynomial, and a 15-points window in a symmetric kernel). Then, several PCAs were performed: one PCA for each individual class (i.e. type of consumer firework), and one overall PCA containing all data. Other specific PCAs containing certain data of interest such as pre-blast or post-blast samples or even postblast samples picked up with a specific swab were useful to solve particular matters. All PCAs were performed using a non-linear iterative partial least squares (NIPALS) algorithm, cross validation with uncertainty test, and 1/SDev as weighing.

\section{Results and discussion}

\subsection{Standards analysis}

First of all, in order to set up a practical compounds library, all the standard reagents of the main compounds found in consumer firework samples were analysed. This library was meant to be useful to subsequently achieve the identification of the compounds contained in the samples. Figure 1a shows the ATR-FTIR spectral signatures of the main 
compounds used to make the selected consumer fireworks such as inorganic salts, charcoal, sulphur or aluminium. Additionally, Figure 1b shows the IR absorption spectra of some possible reaction products, which were selected based on previous works analysing post-blast residues of consumer fireworks [5, 15].
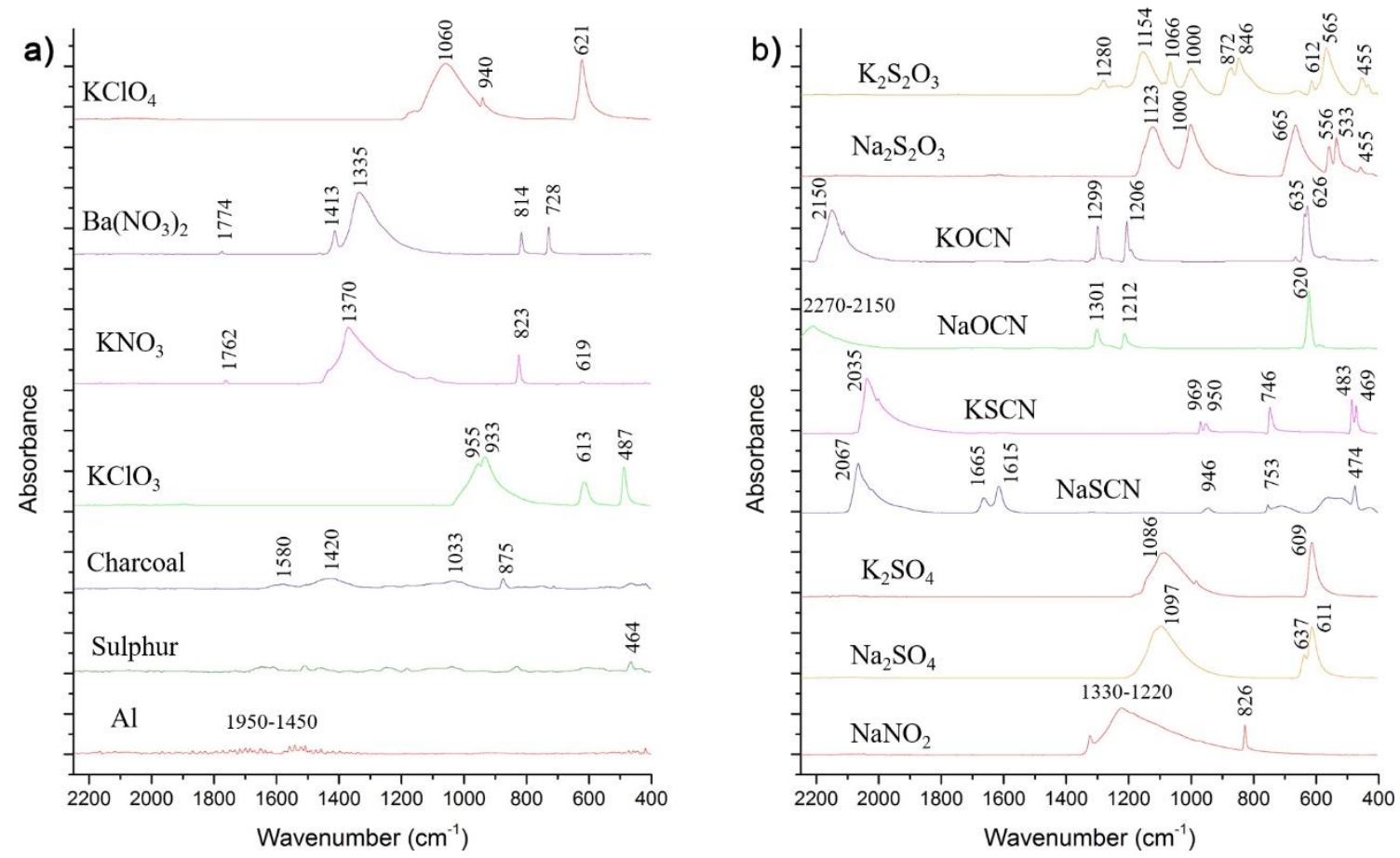

Figure 1. ATR-FTIR absorption spectra for: a) standard reagents usually found in pyrotechnic mixtures of consumer fireworks, b) standard reagents usually found in consumer fireworks postblast residues.

Table 2 shows the spectral information observed in Figure 1, describing the main bands of each compound. The bands were assigned to anion molecular vibrations. In addition, there were some bands which could not be assigned to this type of vibrations. They might be related with the molecular vibrations in the crystal structure of the solid. These bands were also collected in Table 2.

Table 2. Summary of the main compounds used to make consumer fireworks, or that are present in their potential reaction products, as well as their IR bands, and the assignments for the fundamental anion vibrations. (asym: antisymmetric, def: deformation, ip: in-plane, oop: out-ofplane, st: stretching, sym: symmetric). ${ }^{*}=$ band no assigned to fundamental anion vibrations.

\begin{tabular}{cccc} 
Compound & Bands $\left(\mathbf{c m}^{-1}\right)$ & Assignment to anion molecular vibrations & Ref. \\
\hline $\mathbf{K C l O}_{4}$ & 1060,940 & $\mathrm{ClO}_{4}$ triply degenerate asym st $\left(\mathrm{v}_{3}\left(\mathrm{~T}_{2}\right)\right)$ & {$[24]$} \\
& 621 & $\mathrm{ClO}_{4}$ triply degenerate def $\left(\mathrm{v}_{4}\left(\mathrm{~T}_{2}\right)\right)$ & $*$ \\
$\mathbf{B a}\left(\mathrm{NO}_{3}\right)_{2}$ & 1774 & $\mathrm{NO}_{3}$ doubly degenerate asym st $\left(\mathrm{v}_{3}\left(\mathrm{E}^{\prime}\right)\right)$ & {$[25-27]$} \\
& 1413,1335 & $\mathrm{NO}_{3}$ oop def $\left(\mathrm{v}_{2}\left(\mathrm{~A}_{2}{ }_{2}\right)\right)$ & \\
& 814 & $\mathrm{NO}_{3}$ doubly degenerate ip def $\left(\mathrm{v}_{4}\left(\mathrm{E}^{\prime}\right)\right)$ &
\end{tabular}




\begin{tabular}{|c|c|c|c|}
\hline \multirow[t]{4}{*}{$\mathrm{KNO}_{3}$} & 1762 & $*$ & {$[25-27]$} \\
\hline & 1370 & $\mathrm{NO}_{3}$ doubly degenerate asym st $\left(\mathrm{v}_{3}\left(\mathrm{E}^{\prime}\right)\right)$ & \\
\hline & 823 & $\mathrm{NO}_{3}$ oop def $\left(\mathrm{u}_{2}\left(\mathrm{~A}_{2}{ }_{2}\right)\right)$ & \\
\hline & 619 & $\mathrm{NO}_{3}$ doubly degenerate ip def $\left(\mathrm{u}_{4}\left(\mathrm{E}^{\prime}\right)\right)$ & \\
\hline \multirow[t]{4}{*}{$\mathrm{KClO}_{3}$} & 955 & $\mathrm{ClO}_{3}$ doubly degenerate asym st $\left(\mathrm{v}_{3}(\mathrm{E})\right)$ & {$[27]$} \\
\hline & 933 & $\mathrm{ClO}_{3}$ sym st $\left(\mathrm{u}_{1}\left(\mathrm{~A}_{1}\right)\right)$ & \\
\hline & 613 & $\mathrm{ClO}_{3} \operatorname{sym} \operatorname{def}\left(\mathrm{u}_{2}\left(\mathrm{~A}_{1}\right)\right)$ & \\
\hline & 487 & $\mathrm{ClO}_{3}$ doubly degenerate asym def $\left(\mathrm{v}_{4}(\mathrm{E})\right)$ & \\
\hline \multirow{3}{*}{$\begin{array}{c}\text { Charcoal } \\
(\mathrm{C} \text { and } \mathrm{CH})\end{array}$} & 1580,1420 & Ar $\mathrm{C}-\mathrm{C}$ aromatic st & {$[25,26]$} \\
\hline & 1033 & C-H oop bend & \\
\hline & 875 & C-H oop bend & \\
\hline Sulphur & 464 & S-S st & {$[27]$} \\
\hline \multirow{8}{*}{$\mathbf{K}_{2} \mathbf{S}_{2} \mathbf{O}_{3}$} & 1280 & $*$ & {$[27]$} \\
\hline & 1154 & $\mathrm{~S}_{2} \mathrm{O}_{3}$ doubly degenerate asym st $\left(\mathrm{v}_{4}(\mathrm{E})\right)$ & \\
\hline & 1066 & $*$ & \\
\hline & 1000 & $\mathrm{~S}_{2} \mathrm{O}_{3} \operatorname{sym}$ st $\left(\mathrm{v}_{1}\left(\mathrm{~A}_{1}\right)\right)$ & \\
\hline & 872,846 & $*$ & \\
\hline & 612 & $\mathrm{~S}_{2} \mathrm{O}_{3} \operatorname{def}\left(\mathrm{U}_{3}\left(\mathrm{~A}_{1}\right)\right)$ & \\
\hline & 565 & $\mathrm{~S}_{2} \mathrm{O}_{3}$ doubly degenerate def ( $\left.\mathrm{u}_{5}(\mathrm{E})\right)$ & \\
\hline & 455 & S-S st $\left(\mathrm{u}_{2}\left(\mathrm{~A}_{1}\right)\right.$ & \\
\hline \multirow[t]{5}{*}{$\mathrm{Na}_{2} \mathrm{~S}_{2} \mathrm{O}_{3}$} & 1123 & $\mathrm{~S}_{2} \mathrm{O}_{3}$ doubly degenerate asym st $\left(\mathrm{v}_{4}(\mathrm{E})\right)$ & {$[27]$} \\
\hline & 1000 & $\mathrm{~S}_{2} \mathrm{O}_{3}$ sym st $\left(\mathrm{u}_{1}\left(\mathrm{~A}_{1}\right)\right)$ & \\
\hline & 665 & $\mathrm{~S}_{2} \mathrm{O}_{3} \operatorname{def}\left(\mathrm{U}_{3}\left(\mathrm{~A}_{1}\right)\right)$ & \\
\hline & 556,533 & $\mathrm{~S}_{2} \mathrm{O}_{3}$ doubly degenerate def ( $\left.\mathrm{u}_{5}(\mathrm{E})\right)$ & \\
\hline & 455 & S-S st $\left(\mathrm{U}_{2}\left(\mathrm{~A}_{1}\right)\right.$ & \\
\hline \multirow[t]{3}{*}{ KOCN } & 2150 & $\mathrm{C} \equiv \mathrm{N}$ st $\left(\mathrm{U}_{1}\right)$ & {$[25,26]$} \\
\hline & 1299,1206 & $\mathrm{O}-\mathrm{C}$ st $\left(\mathrm{u}_{3}\right)$ & \\
\hline & 635,626 & $\mathrm{O}-\mathrm{C} \equiv \mathrm{N}$ def $\left(\mathrm{U}_{2}\right)$ & \\
\hline \multirow[t]{3}{*}{$\mathrm{NaOCN}$} & $2270-2150$ & $\mathrm{C} \equiv \mathrm{N}$ st $\left(\mathrm{v}_{1}\right)$ & {$[25,26]$} \\
\hline & 1301,1212 & $\mathrm{O}-\mathrm{C}$ st $\left(\mathrm{v}_{3}\right)$ & \\
\hline & 620 & $\mathrm{O}-\mathrm{C} \equiv \mathrm{N}$ def $\left(\mathrm{u}_{2}\right)$ & \\
\hline \multirow[t]{4}{*}{ KSCN } & 2035 & $\mathrm{C} \equiv \mathrm{N}$ st $\left(\mathrm{v}_{1}\right)$ & {$[25,27]$} \\
\hline & 969,950 & $*$ & \\
\hline & 746 & S-C st (u3) & \\
\hline & 483,469 & $\mathrm{~S}-\mathrm{C} \equiv \mathrm{N} \operatorname{def}\left(\mathrm{u}_{2}\right)$ & \\
\hline \multirow[t]{5}{*}{$\mathrm{NaSCN}$} & 2067 & $\mathrm{C} \equiv \mathrm{N}$ st $\left(\mathrm{u}_{1}\right)$ & {$[26,27]$} \\
\hline & 1665,1615 & $*$ & \\
\hline & 946 & * & \\
\hline & 753 & $\mathrm{~S}-\mathrm{C}$ st $\left(\mathrm{u}_{3}\right)$ & \\
\hline & 474 & $\mathrm{~S}-\mathrm{C} \equiv \mathrm{N} \operatorname{def}\left(\mathrm{u}_{2}\right)$ & \\
\hline \multirow[t]{2}{*}{$\mathrm{K}_{2} \mathrm{SO}_{4}$} & 1086 & $\mathrm{SO}_{4}$ triply degenerate asym st $\left(\mathrm{u}_{3}\left(\mathrm{~T}_{2}\right)\right)$ & {$[25]$} \\
\hline & 609 & $\mathrm{SO}_{4}$ triply degenerate $\operatorname{def}\left(\mathrm{U}_{4}\left(\mathrm{~T}_{2}\right)\right)$ & \\
\hline \multirow[t]{2}{*}{$\mathrm{Na}_{2} \mathrm{SO}_{4}$} & 1097 & $\mathrm{SO}_{4}$ triply degenerate asym st $\left(\mathrm{v}_{3}\left(\mathrm{~T}_{2}\right)\right)$ & {$[27]$} \\
\hline & 637,611 & $\mathrm{SO}_{4}$ triply degenerate $\operatorname{def}\left(\mathrm{v}_{4}\left(\mathrm{~T}_{2}\right)\right)$ & \\
\hline \multirow[t]{3}{*}{$\mathrm{NaNO}_{2}$} & 1330 & $\mathrm{NO}_{2}$ sym st $\left(\mathrm{v}_{1}\left(\mathrm{~A}_{1}\right)\right)$ & {$[25,27]$} \\
\hline & 1220 & $\mathrm{NO}_{2}$ asym st $\left(\mathrm{v}_{3}\left(\mathrm{~B}_{2}\right)\right)$ & \\
\hline & 826 & $\mathrm{NO}_{2} \operatorname{def}\left(\mathrm{u}_{2}\left(\mathrm{~A}_{1}\right)\right)$ & \\
\hline
\end{tabular}

As can be seen in Figure 1a, aluminium showed an interesting IR absorption spectrum. It displayed several signals at approximately 1950-1450 $\mathrm{cm}^{-1}$. However, there is not molecular vibrations in this element that can explain these signals. When another ATRFTIR instrument was employed for the analysis of the same sample, the same result was obtained. However, when aluminium is measured by transmission using $\mathrm{KBr}$ pellet 
method instead of ATR, these signals do not appear (data not shown). Therefore, this effect might be produced only when using the ATR-FTIR measurements which could be explained by some kind of reflected radiation caused by the aluminium in contact with the ATR crystal, although further research would be necessary to confirm that. Nonetheless, the spectrum obtained by ATR-FTIR might be useful for the identification of this element, although its signal is not explained by its vibrational properties.

Additionally, Figure S1 (supplementary material) shows the IR absorption spectra of other compounds less frequently used in consumer fireworks.

\subsection{Sample analysis}

Five consumer fireworks available in the Spanish market were analysed: four different firecrackers (named as F1, F2, F3, and F4), and one rocket (named as R1). F1, F2 and F3 are simple firecrackers which have a fuse and a charge, and F4 is a complex firecracker which have a fuse and several charges. The simple and fast procedure for obtaining spectra of post-blast residues from consumer fireworks started by swabbing the surface of interest with dry cotton swabs. Then, the swabs were directly placed and pressed on the diamond crystal surface of the ATR-FTIR device. Therefore, first, these items were lit, and after they went off, their post-blast residues were swabbed and analysed. Other consumer fireworks of the same characteristics were also selected and dissected separately for the analysis of their unburnt fuses and unexploded charges.

The F1 item was exploded on a steel plate and its residues were swabbed. Most of the original reagents usually turn into gaseous products such as $\mathrm{CO}_{2}$ and $\mathrm{H}_{2} \mathrm{O}$. However, part of the total charge may remain unburnt. Figure 2 shows the F1 post-blast spectrum in which weak signals appear at around $1950-1450 \mathrm{~cm}^{-1}$. These signals might correspond to Al. It also shows several bands spread over a wide range at approximately $1170-930 \mathrm{~cm}^{-1}$, and a sharp band at around $621 \mathrm{~cm}^{-1}$. These bands might be due to the presence of $\mathrm{KClO}_{4}$. Both, $\mathrm{Al}$ and $\mathrm{KClO}_{4}$ are part of the initial components of this item (Table 1). Furthermore, the bands observed at around 1450-1200 $\mathrm{cm}^{-1}$ and the small peak at $823 \mathrm{~cm}^{-1}$ match up with the $\mathrm{KNO}_{3}$ spectrum. Therefore, those bands seem to indicate the presence of this salt in the F1 post-blast residues. Additional bands appeared in this spectrum at around 2165 and $2065 \mathrm{~cm}^{-1}$. According to the bibliography and our IR spectral library (Table 2), these bands might correspond to vibrations of cyanate and thiocyanate groups. Therefore, these compounds may belong to the combustion products from the "explosion" of this 
pyrotechnic composition [5]. For instance, thiocyanate is produced from sulphur in the presence of nitrogenous compounds. Besides, some of those $\mathrm{KClO}_{4}$ related bands (at around 1170-930 and $621 \mathrm{~cm}^{-1}$ ), could be overlapping with other bands of sulphur reaction products such as sulphate or thiosulphate. A double band at around $880-860 \mathrm{~cm}^{-1}$ and bands at approximately 668 and $524 \mathrm{~cm}^{-1}$ might also correspond to potassium or sodium thiosulphate salts. Both thiosulphate salts could be produced by combustion because both cations are already present in the item, potassium as $\mathrm{KNO}_{3}$, and sodium in the clay plugs of this item [15].

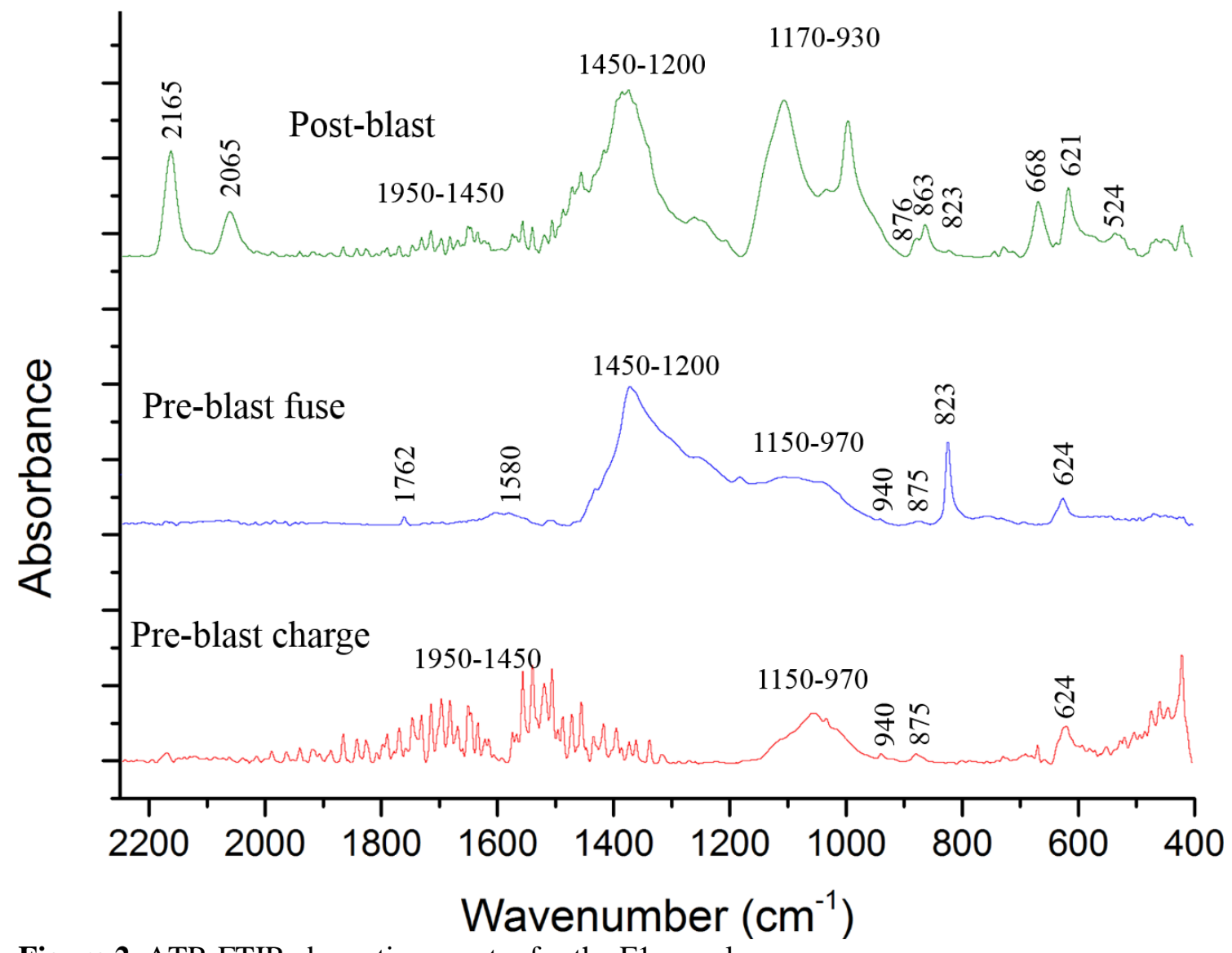

Figure 2. ATR-FTIR absorption spectra for the F1 samples.

It is important to note that the bands showed in the spectrum might be related to other reaction products or from cross-contamination, or from additional compounds contained in the item clay plugs or cartridge. Then, pre-blast analysis of the F1 fuse and charge were carried out in order to compare their spectra with the post-blast spectrum (Figure 2). In the pre-blast fuse, characteristic IR bands of $\mathrm{KNO}_{3}$ (at about 1762, 823, and 1450-1200 $\mathrm{cm}^{-1}$ ), and some weak bands at about 1150-970, 940, and $624 \mathrm{~cm}^{-1}$ were detected. These last bands might correspond to the presence of perchlorate salt. In a previous work by CE, nitrate and perchlorate were identified in the F1 fuse [5]. Besides, some bands that might 
be related to charcoal (i.e., 1580 and $875 \mathrm{~cm}^{-1}$ ) were also identified. In the pre-blast F1 charge, the unusual signal corresponding to $\mathrm{Al}$ (at about 1950-1450 cm $\mathrm{cm}^{-1}$ ) was detected, as well as very weak bands at approximately 1150-970, 940 and at $624 \mathrm{~cm}^{-1}$, which might correspond to vibrations of the perchlorate anions. In the light of these results, it seems that this procedure makes it possible to trace the original fireworks composition in their post-blast residues. F1 might be made with a Pyrodex (mixture of $\mathrm{KClO}_{4}, \mathrm{KNO}_{3}$, charcoal, sulphur, and other reagents) fuse, and a flash powder $\left(\mathrm{KClO}_{4}\right.$ and $\left.\mathrm{Al}\right)$ charge.

Figure 3 shows the IR spectra for the F2 post-blast residues, and fuse and charge preblast samples. The F2 post-blast residues spectrum displayed some bands similar to those of F1 post-blast residues. This suggests a composition with analogous unburnt reagents (nitrate and perchlorate salts, and aluminium), and reaction products (cyanate, thiocyanate, and sulphate salts). In fact, both items are very similar in their declared composition (Table 1). Regarding the pre-blast fuse spectrum, it showed bands related to $\mathrm{KNO}_{3}$ (at around 1762, 1375, and $823 \mathrm{~cm}^{-1}$ ). Besides, some bands possibly related to charcoal are also displayed: a broad band at around $1033 \mathrm{~cm}^{-1}$, and additional bands at approximately 1580 and $875 \mathrm{~cm}^{-1}$. Although, the band at around $1033 \mathrm{~cm}^{-1}$ could also be related to perchlorate, the typical sharp band of $\mathrm{KClO}_{4}$ at $621 \mathrm{~cm}^{-1}$ did not appear. In the analysis by CE, this fuse only showed nitrate anions [5]. Finally, this fuse also showed a band at around $465 \mathrm{~cm}^{-1}$ which could be related to sulphur. Regarding the pre-blast charge spectrum, it showed the signal related to $\mathrm{Al}$ at approximately $1950-1450 \mathrm{~cm}^{-1}$. The very broad bands at around 1150-930 and at $621 \mathrm{~cm}^{-1}$ could also indicate the presence of perchlorate. Hence, F2 might be made off a black powder $\left(\mathrm{KNO}_{3}\right.$, sulphur, and charcoal) fuse, and a flash powder charge. 


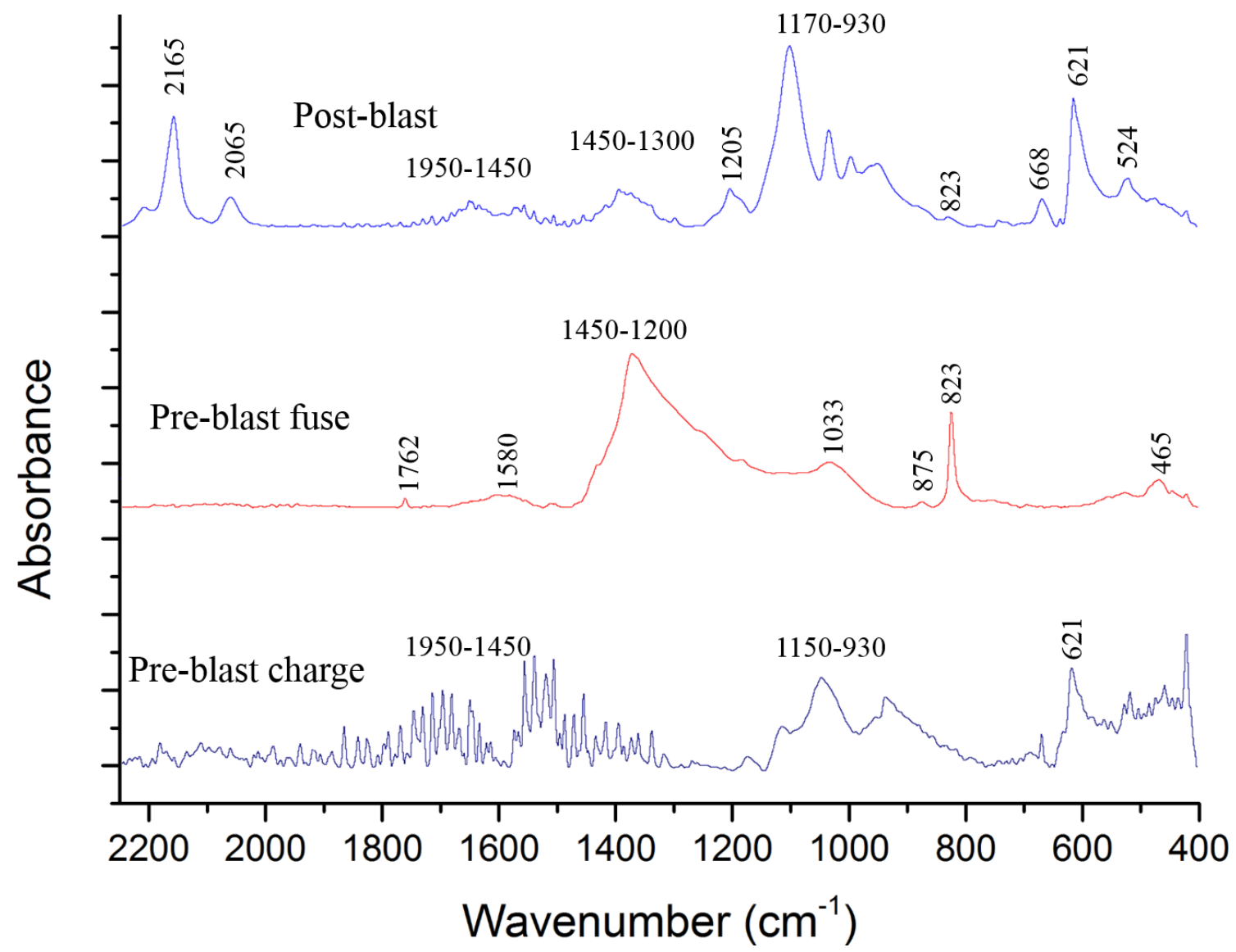

Figure 3. ATR-FTIR absorption spectra for the F2 samples.

Figure 4 shows the spectra of F3 post-blast residues, pre-blast fuse, and pre-blast charge samples. In the F3 post-blast residues, some detected bands might correspond to initial compounds such as $\mathrm{Al}$ (at about 1950-1450 $\mathrm{cm}^{-1}$ ), and $\mathrm{KClO}_{4}$ (at about 1170-930, and $621 \mathrm{~cm}^{-1}$ ). Moreover, other identified bands might be related to nitrate salts (at about $1450-1325 \mathrm{~cm}^{-1}$ ), and reaction products such as sulphate or thiosulphate (at approximately 1170-930, 668, and $524 \mathrm{~cm}^{-1}$ ), and cyanate and thiocyanate salts (at about 1025 and above $2000 \mathrm{~cm}^{-1}$ ). The pre-blast fuse spectrum clearly showed bands corresponding to a nitrate salt (at about 1762, 1450-1200, and $823 \mathrm{~cm}^{-1}$ ), and strong bands related to perchlorate salt (at approximately $1170-930$, and $621 \mathrm{~cm}^{-1}$ ). These compounds seem to be part of the composition of this type of fuse. Furthermore, the small band at around $465 \mathrm{~cm}^{-1}$ and another weak band at about $875 \mathrm{~cm}^{-1}$ might indicate the presence of sulphur and charcoal. The pre-blast charge spectrum showed bands related to $\mathrm{Al}$ and $\mathrm{KClO}_{4}$, which agree with the results achieved by CE [5]. However, the pre-blast charge spectrum showed also a band at approximately $875 \mathrm{~cm}^{-1}$ which could be related to charcoal from crosscontamination between fuse and charge. Hence, F3 might be made off with a Pyrodex fuse and a flash powder charge. 


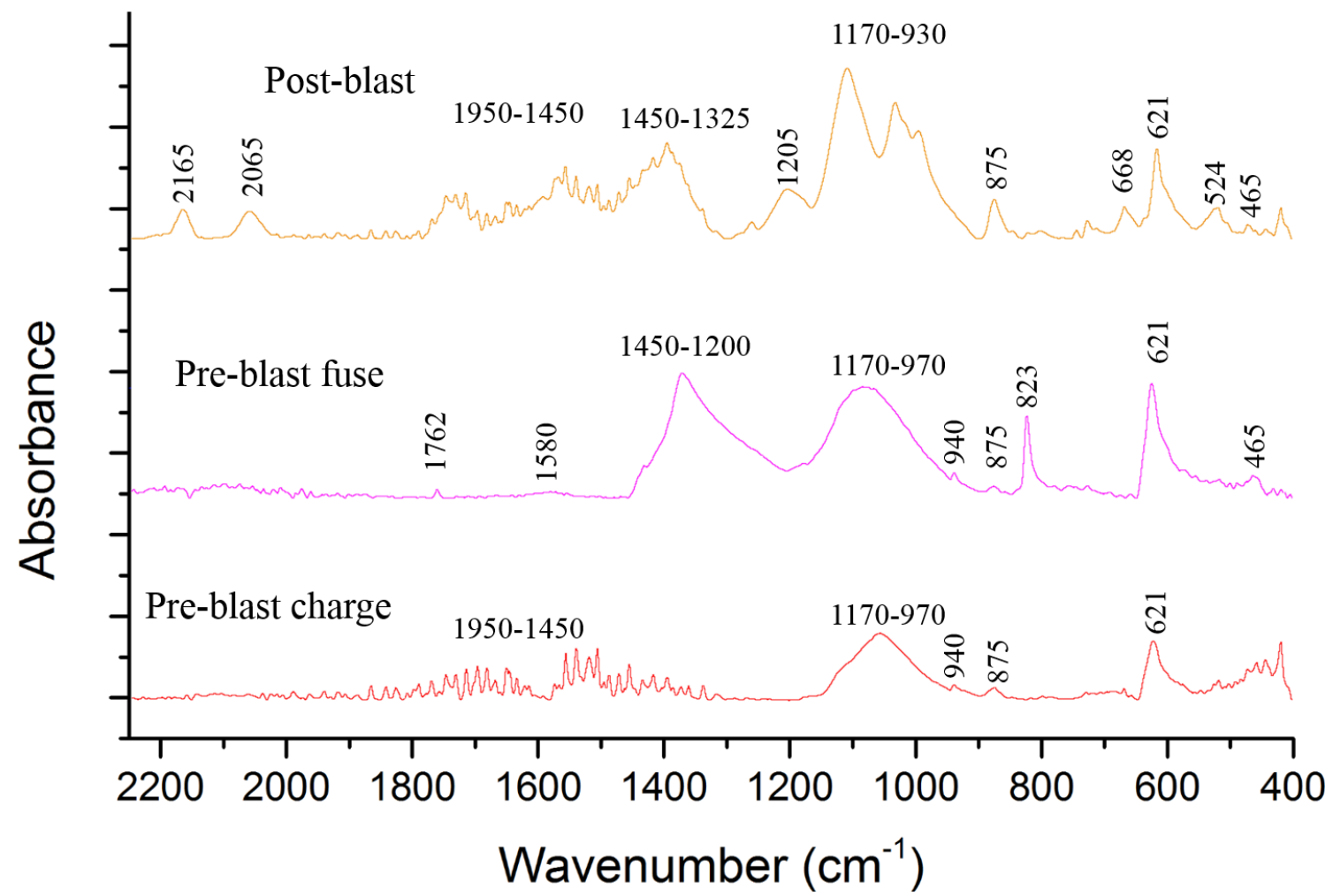

Figure 4. ATR-FTIR absorption spectra for the F3 samples.

F4 is a complex firecracker, having three different charges in the same compartment; one after another. These charges produce different pyrotechnic effects (smoke and cracking, and a final thunder), and have different chemical compositions. An item was exploded, and its post-blast residues were analysed. Figure 5 shows the spectra of the F4 post-blast residues, pre-blast fuse and thunder charges, and pre-blast smoke and cracking charges samples. The spread signal appearing at about $1950-1450 \mathrm{~cm}^{-1}$ may correspond to $\mathrm{Al}$, while the bands at approximately $1170-930 \mathrm{~cm}^{-1}$ might be related to $\mathrm{KClO}_{4}$. The spectra also shows some weak bands related to combustion products like sulphate or thiosulphate (at around 1170-930, 668, and $524 \mathrm{~cm}^{-1}$ ), and cyanate and thiocyanate (at about 1205, 2065, and 2230-2150 $\mathrm{cm}^{-1}$ ). Another broad band was also observed at around $905 \mathrm{~cm}^{-1}$, and could correspond to $\mathrm{KClO}_{3}$, although this compound was not declared by the manufacturers probably because its use in this type of mixtures is banned in some countries [5]. Additionally, a sharp band observed at about $613 \mathrm{~cm}^{-1}$ may also be related to the initial compounds (e.g., $\mathrm{KClO}_{4}$ or $\mathrm{KClO}_{3}$ ) or to several reaction products (sulphate, thiosulphate or cyanate). 


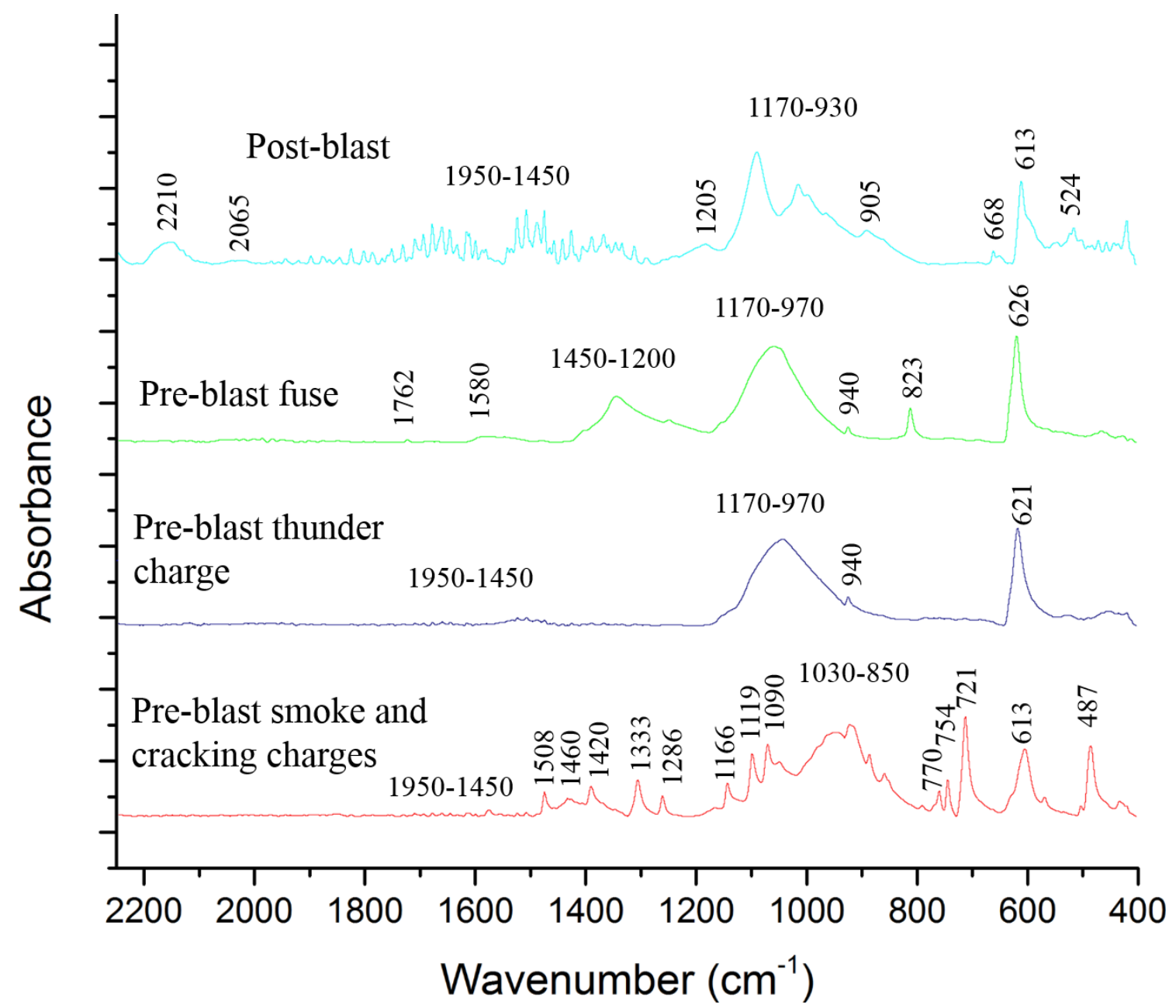

Figure 5. ATR-FTIR absorption spectra for the F4 samples.

Regarding the pre-blast fuse spectrum, it displayed bands corresponding to $\mathrm{KNO}_{3}$ (at about 1762, 1450-1200, and $823 \mathrm{~cm}^{-1}$ ), and $\mathrm{KClO}_{4}$ (at around 1170-970, 940 and 642$593 \mathrm{~cm}^{-1}$ ), which agrees with previous findings $[5,15]$. A weak band observed at about $1580 \mathrm{~cm}^{-1}$ could be related to the presence of charcoal. Regarding the pre-blast thunder effect, and the smoke and cracking mixtures, there was no way to completely separate the smoke and cracking pyrotechnic effect mixtures without causing cross-contamination. Consequently, both mixtures were analysed together, while the thunder effect charge was analysed separately. The thunder effect mixture displayed the signal of $\mathrm{Al}$, and bands at about 1060, 940, and $621 \mathrm{~cm}^{-1}$, which might be related to $\mathrm{KClO}_{4}$. The mixture of the other two powders showed bands at about 487 , and $613 \mathrm{~cm}^{-1}$, and in the $1030-850 \mathrm{~cm}^{-1}$ range, which might correspond to $\mathrm{KClO}_{3}$. As was suspected after analysing its post-blast spectrum, several bands suggest the presence of $\mathrm{KClO}_{3}$ although it was not declared by the manufacturers. Furthermore, it was not a surprise to find chlorate anions in this type of firecracker $[5,15]$. Although, other bands were also observed in this spectrum, they could not be properly assigned. Some of them might correspond to the "inert material" 
declared by the manufacturers. Hence, F4 seems to be composed of a fuse probably made of Pyrodex $\left(\mathrm{KNO}_{3}\right.$ and $\left.\mathrm{KClO}_{4}\right)$, and several charges. One of the charges might be flash powder (thunder effect powder), and the smoke effect powder might contain $\mathrm{KClO}_{3}$ as part of its composition.

$\mathrm{R} 1$ is a bottle rocket, a complex item with several charges and an external, and another internal, fuse. Their charge composition is as follows: a lift mixture, a burst mixture, and a main charge with the final effect. Figure $\mathbf{6}$ shows the spectra of post-blast residues, and pre-blast samples of the external fuse, the burst and lift mixtures, and the main charge of the bottle rocket. The spectrum of the post-blast residues showed signals that might be related to $\mathrm{Al}$ (at about $1950-1450 \mathrm{~cm}^{-1}$ ), and bands related to $\mathrm{KClO}_{4}$ (at approximately 1170-930, and $619 \mathrm{~cm}^{-1}$ ) and related to combustion products like thiocyanate (above 2000 $\mathrm{cm}^{-1}$ ) or sulphate (at around 1170-930, and $670 \mathrm{~cm}^{-1}$ ). Another R1 item was also dissected and its parts analysed. The bands found in the pre-blast fuse analysis might be related to $\mathrm{KClO}_{4}$ (at about 1060, 940, and $621 \mathrm{~cm}^{-1}$ ). In addition, several bands appeared spread over the spectrum, but their corresponding compounds were not identified, despite that perchlorate was found in a previous work [5]. The burst and lift mixtures showed bands that might correspond to $\mathrm{KNO}_{3}$ (at about 1762, 1450-1200, and $823 \mathrm{~cm}^{-1}$ ). Finally, the pre-blast spectrum of the final effect composition showed bands that could be related to $\mathrm{Al}, \mathrm{Ba}\left(\mathrm{NO}_{3}\right)_{2}$ (at about 1413 and $1340 \mathrm{~cm}^{-1}$, and sharp bands at 814 and $728 \mathrm{~cm}^{-1}$ ), and $\mathrm{KClO}_{4}$ (at about 1170-930, and $623 \mathrm{~cm}^{-1}$ ). These results match with our-previous findings $[5,15]$. Hence, the bottle rocket seems to be composed by a fuse containing $\mathrm{KClO}_{4}$, a body with lift and burst mixtures made off black powder, and a final pyrotechnic effect containing a complex green colouring mixture with $\mathrm{Al}, \mathrm{Ba}\left(\mathrm{NO}_{3}\right)_{2}$ and $\mathrm{KClO}_{4}$. 


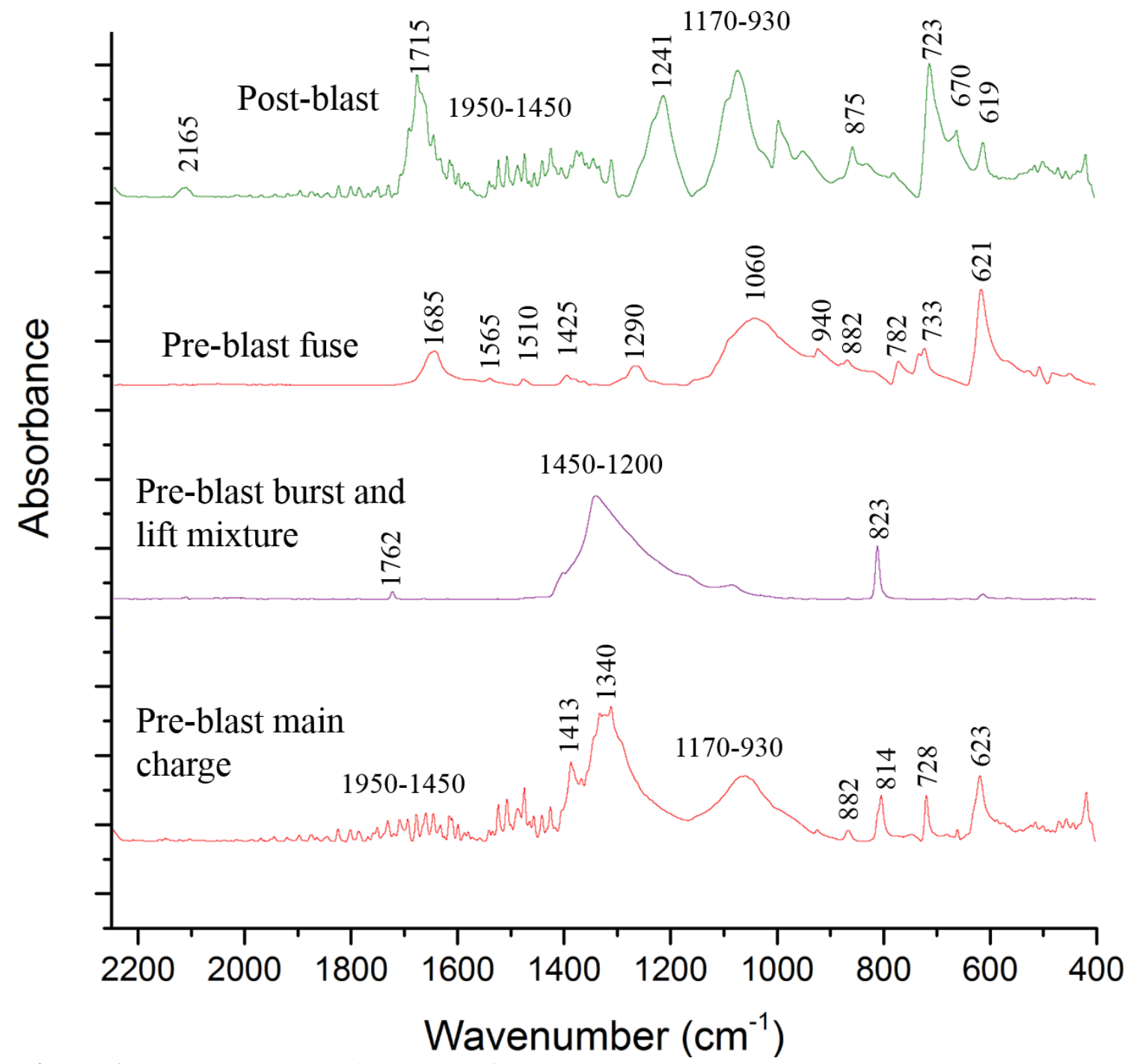

Figure 6. ATR-FTIR absorption spectra for the R1 samples.

Additionally, for each item discussed above, those areas in which there were only either fuse or charge residues where also swabbed. There existed differences between these spectra and the spectrum obtained when swabbing residues from all over the surface. Nevertheless, the sum of these two independent spectra sets resulted in almost the whole post-blast spectrum showed and discussed above.

\subsection{Swabbing, a practical aspect to be considered}

In real cases, the residues amount or the type of collection surfaces are normally unknown variables, and so the residues collection is a crucial factor to be evaluated carefully. In that context, the swab head type/material has to be considered a critical issue during evidence collection [28]. The challenge here is two-fold: the effectivity of the swabbing system on different surfaces (physical aspect), and the background signals produced by 
the swab head material (chemical aspect). In this work, the post-blast residues were collected using three different dry cotton swabs named as $\mathrm{Cb}, \mathrm{Sw}$, and $\mathrm{Hy}$. The swabs were analysed before any swabbing (blank samples), giving several weak bands in their spectral fingerprint region. The $\mathrm{Sw}$ swab showed quite similar signals as the $\mathrm{Cb}$ swab, thus indicating they were made off with almost the same type of material. On the contrary, when post-blast residues were swabbed and analysed, the weak in-the-blank bands did not appear. Nonetheless, in a few occasions, and regardless of the swab type, some of the cotton head bands appeared in the spectrum whenever the amount of collected residues was not enough to cover the cotton head.

Therefore, this work used chemometric techniques for studying a firecracker as a model, focusing on the effectivity of the swabbing procedure using three different swabs on two different surfaces. Two F4 items were exploded on a steal surface (Ms) and a glass surface (Des). The steal surface was an open metallic sheet, while the other surface was an uncovered large glass desiccator. In both cases, a relatively good amount of post-blast residues was available, although the amount of residues is assumed higher in the case of the glass desiccator because of a lower dispersion of the residues. Post-blast residues were collected using three different swabs, gathering residues from all the surface. In addition, only samples from the fuse residues area and from the item body area were taken. In order to avoid redundancy and overcrowding, the $\mathrm{Sw}$ and $\mathrm{Cb}$ spectra were not plotted because they were quite similar. Figure 7a shows a basic 2D PCA Scores plot performed on the post-blast F4 residues collected on two surfaces (Ms and Des) with two types of swabs ( $\mathrm{Cb}$ and $\mathrm{Hy}$ ). Figure $\mathbf{7 b}$ shows a basic 3D PCA Scores plot with the same features as the 2D Scores plot.
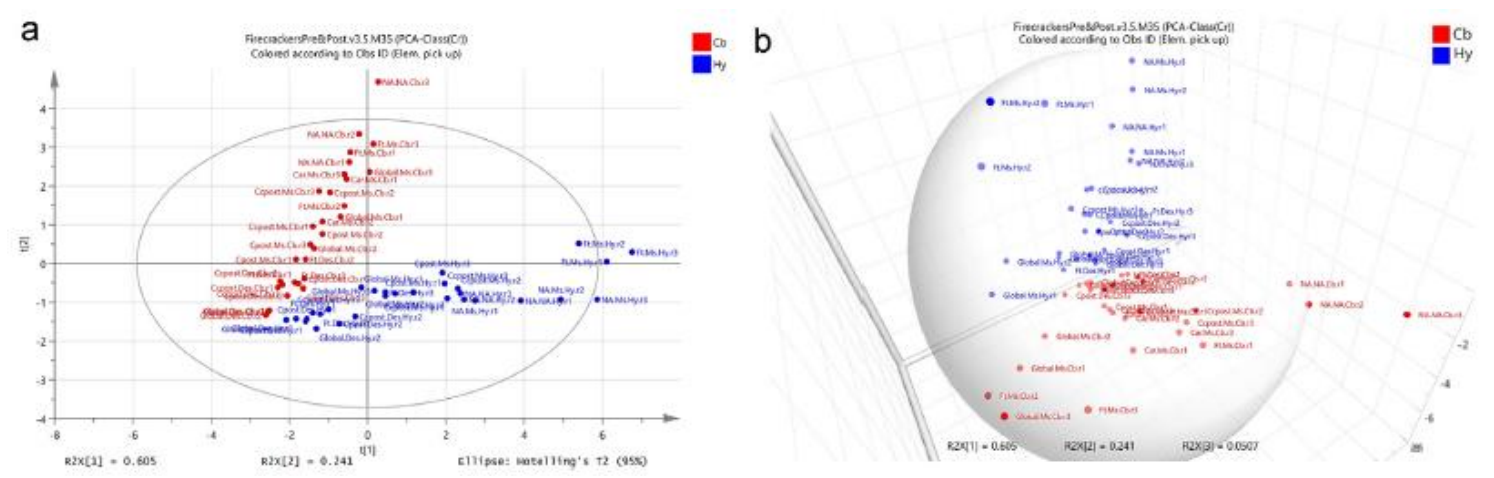

Figure 7. (a) Basic 2D and, (b) 3D PCA Scores plots for the post-blast crack firecracker residues collected from two surfaces (Ms and Des) with two types of swabs (Cb and Hy). 
As it was expected, the results indicated that the most important sources of variability are indeed the two collection devices (i.e., swab types), and the post-blast residues concentration (Figure 7a). The planes of the two swab types tend to converge at certain point on the left-hand side of the Scores plot. This suggests that the influence of the collection device gradually decreases when the post-blast residues concentration increases (as inside the desiccator). On the contrary, the importance of the collection device increases with the combination of two factors (Figure 7b). First, with lower postblast residues concentration (as on the metallic sheet), moving towards the ultimate blankswab behaviour. Second, with higher total fuse concentration, showing that the post-blast fuse components were highly concentrated when collected, and that they are rather different compared to the post-blast charge components. Nevertheless, it is clear for the F4 item that the results are very different depending on the swab used for the collection. This behaviour varied with the collection place, which has to do with the post-blast residues concentration (especially for the fuse residues), and the part of the firecracker being analysed. Although the methodology proposed in this work was suitable for studying consumer fireworks post-blast residues under the above mentioned experimental conditions, more research is needed in order to find an effective collection device useful for a wide variety of surfaces.

\section{Conclusions}

In this work, the acquisition of IR absorption spectra from consumer firework post-blast residues was achieved by means of a simple and fast sampling procedure and subsequent analysis by ATR-FTIR.

The acquired post-blast spectra made it possible to trace the post-blast residues back to the original pyrotechnic compositions. This way, the items F1, F2 and F3 were composed by $\mathrm{Al}, \mathrm{KClO}_{4}, \mathrm{KNO}_{3}$ and sulphur. In the complex $\mathrm{F} 4$ consumer firework, $\mathrm{Al}, \mathrm{KClO}_{4}$, $\mathrm{KClO}_{3}$, and sulphur were detected. In the same way, a nitrate salt was identified by the presence of its reaction products. The rocket $\mathrm{R} 1$ showed $\mathrm{Al}, \mathrm{KClO}_{4}$, sulphur, and the presence of nitrate reaction products. Furthermore, this work shows that the analysis of pre-blast items by ATR-FTIR can provide additional information about the composition of each part. Different fuse compositions were identified in the selected items. Moreover, F4 showed $\mathrm{KClO}_{3}$ in its smoke pyrotechnic mixture, which is a compound banned in 
some countries. At the same time, $\mathrm{R} 1$ showed $\mathrm{Ba}\left(\mathrm{NO}_{3}\right)_{2}$ in one of its charges. An important issue to highlight is the apparition of several signals at approximately 1950$1450 \mathrm{~cm}^{-1}$ when $\mathrm{Al}$ is analysed by ATR-FTIR. These signals can not be explained by the vibrational properties of $\mathrm{Al}$, although empirically showed their usefulness to determine the presence of $\mathrm{Al}$ in this work. Further research would be necessary to better understand this effect.

This study also found that few analysis showed several weak background bands spread through the fingerprint region, probably because the swabbing procedure was not $100 \%$ effective in those cases. Therefore, this work studied one firecracker as a model and focused on the effectivity of the swabbing system on two surfaces using three different cotton swabs. Using chemometric techniques it was found that the results were very different depending on the swab used for the residues collection and on the collection place, which relates to the post-blast residues concentration. Although this methodology was suitable to study post-blast residues from consumer fireworks over some surfaces, more research is necessary for the purpose of finding an appropriate collection device for sampling a wider variety of substrates. This work might be the first step towards proposing ATR-FTIR spectroscopy as complementary technique for the analysis of postblast residues from consumer fireworks.

\section{Acknowledgments}

The authors would like to greatly thank Carlos Villar (Bruker Optics Inc., Spain) for facilitating the ATR-FTIR system. Carlos Martín-Alberca and Félix Zapata thank the University of Alcalá for their pre-doctoral grants. We also would like to thank I. Jimeno for the graphic arts. 


\section{References}

[1] C. Martín-Alberca, C. García-Ruiz, Analytical techniques for the analysis of consumer fireworks, Trends Anal. Chem. 54 (2014) 27-36.

[2] J. A. Conkling, C. Mocella, Chemistry of Pyrotechnics. Basic Principles and Theory, CRC Press, Taylor \& Francis Inc, Boca Raton, FL, Second Ed. 2011.

[3] Y. Tu, D.V. Granados, Fireworks Annual Report: Fireworks-Related Deaths, Emergency Department-Treated Injuries, and Enforcement Activities During 2013, U.S. Consumer Product Safety Commission, Washington, DC, 2013

[4] TWGFEX (Technical Working Group for Fire and Explosives), Laboratory Explosion Group: Standards \& Protocols Committee, Recommended Guidelines for Forensic Identification of Post-Blast Explosive Residues.

[5] C. Martín-Alberca, M.Á. Fernández de la Ossa, J. Sáiz, J.L. Ferrando, C. García-Ruiz, Anions in pre- and post-blast consumer fireworks by capillary electrophoresis, Electrophoresis 35 (2014) 3272-3280.

[6] P. Alenfelt, Chemical analysis of consumer fireworks, J. Pyrotech. 11 (2000) 11-15.

[7] D. Chapman, Techniques for the quantitative analysis of sulfur and chlorate in fireworks compositions, J. Pyrotech. 5 (1997) 25-32.

[8] S.A. Phillips, Pyrotechnic residues analysis - detection and analysis of characteristic particles by scanning electron microscopy/energy dispersive spectroscopy, Sci. Justice 41 (2001) 73-80.

[9] K.L. Kosanke, R.C. Dujay, B.J. Kosanke, Characterization of pyrotechnic reaction residue particles by SEM/EDS, J. Forensic Sci. 48 (2003) 531-537.

[10] K.L. Kosanke, R.C. Dujay, B.J. Kosanke, Pyrotechnic reaction residue particle analysis, J. Forensic Sci. 51 (2006) 296-302.

[11] M. Grima, M. Butler, R. Hanson, A. Mohameden, Firework displays as sources of particles similar to gunshot residue, Sci. Justice 52 (2012) 49-57. 
[12] T. Kishi, J. Nakamura, H. Arai, Application of capillary electrophoresis for the determination of inorganic ions in trace explosives and explosive residues, Electrophoresis 19 (1998) 3-5.

[13] K.G. Hopper, H. LeClair, B.R. McCord, A novel method for analysis of explosives residue by simultaneous detection of anions and cations via capillary zone electrophoresis, Talanta 67 (2005) 304-312.

[14] C. Sarazin, N. Dalaunay, C. Costanza, V. Eudes, P. Gareil, Capillary electrophoresis analysis of inorganic cations in post-blast residue extracts applying a guanidinium-based electrolyte and bilayer-coated capillaries, Electrophoresis 32 (2011) 1282-1291.

[15] J. Sáiz, M.T. Duc, I.J. Koenka, C. Martín-Alberca, P.C. Hauser, C. García-Ruiz, Concurrent determination of anions and cations in consumer fireworks with a portable dual-capillary electrophoresis system, J. Chromatogr. A, 1372 (2014) 245-252.

[16] R.K. Wharton, D. Chapman, A.E. Jeffcock, Evaluation of the hazards posed by high energy bangers Part 1. Noise, overpressure and TNT equivalence, J. Pyrotech. 15 (2002) $1-8$.

[17] E. Sokol, A.U. Jackson, R.G. Cooks, Trace detection of inorganic oxidants using desorption electrospray ionization (DESI) mass spectrometry, Cent. Eur. J. Chem. 9 (2011) 790-797.

[18] P.M. Flanigan, J.J. Brady, E.J. Jude, R.J. Levis, Determination of inorganic improvised explosive device signatures using laser electrospray mass spectrometry detection with offline classification, Anal. Chem. 83 (2011) 7115-7122.

[19] M. López-López, C. García-Ruiz, Infrared and Raman spectroscopy techniques applied to identification of explosives, Trends Anal. Chem. 54 (2014) 36-44.

[20] K. Castro, S. Fdez-Ortiz de Vallejuelo, I. Astondoa, F.M. Goñi, J.M. Madariaga, Analysis of confiscated fireworks using Raman spectroscopy assisted with SEM-EDS and FTIR, J. Raman Spectrosc. 42 (2011) 2000-2005.

[21] K. Szomborg, F. Jongekrijg, E. Gilchrist, T. Webb, D. Wood, L. Barron, Residues from low-order energetic materials: the comparative performance of a range of sampling approaches prior to analysis by ion chromatography, Forensic Sci. Int. 233 (2013) 55-62. 
[22] D.A. DeTata, A.P. Collins, J.A. McKinley, A comparison of common swabbing materials for the recovery of organic and inorganic explosive residues, J. Forensic Sci., 58,3 (2013) 757-763.

[23] N. Song-im, S. Benson, C. Lennard, Establishing a universal swabbing and clean-up protocol for the combined recovery of organic and inorganic explosive residues, Forensic Sci. Int. 223 (2012) 136-147.

[24] G. Raj, A. Bhagi, V. Jain, Group theory and Symmetry in Chemistry, Krishna Prakashan Media Ltd., Meerut, 1998.

[25] D.W. Mayo, F.A. Miller, R.W. Hannah. "Course Notes on the Interpretation of Infrared and Raman Spectra", Chapter 11 "Infrared spectra of inorganic materials" John Wiley \& Sons, Inc., Hoboken, New Jersey, 2003 pp. 297-354.

[26] Richard A. Nyquist, Ronald O. Kagel, Handbook of Infrared and Raman Spectra of Inorganic Compounds and Organic Salts. Chapter 1 "Infrared Spectra of Inorganic Compounds", 1971 pp. 1-18.

[27] K. Nakamoto, Infrared and Raman spectra of inorganic and coordination compounds, Part A. Theory and applications in inorganic chemistry. 6th edition, John Wiley and Sons Inc., New York 2009.

[28] N. Song-im, S. Benson, C. Lennard, Evaluation of different sampling media for their potential use as a combined swab for the collection of both organic and inorganic explosive residues, Forensic Sci. Int. 222 (2012) 102-11. 


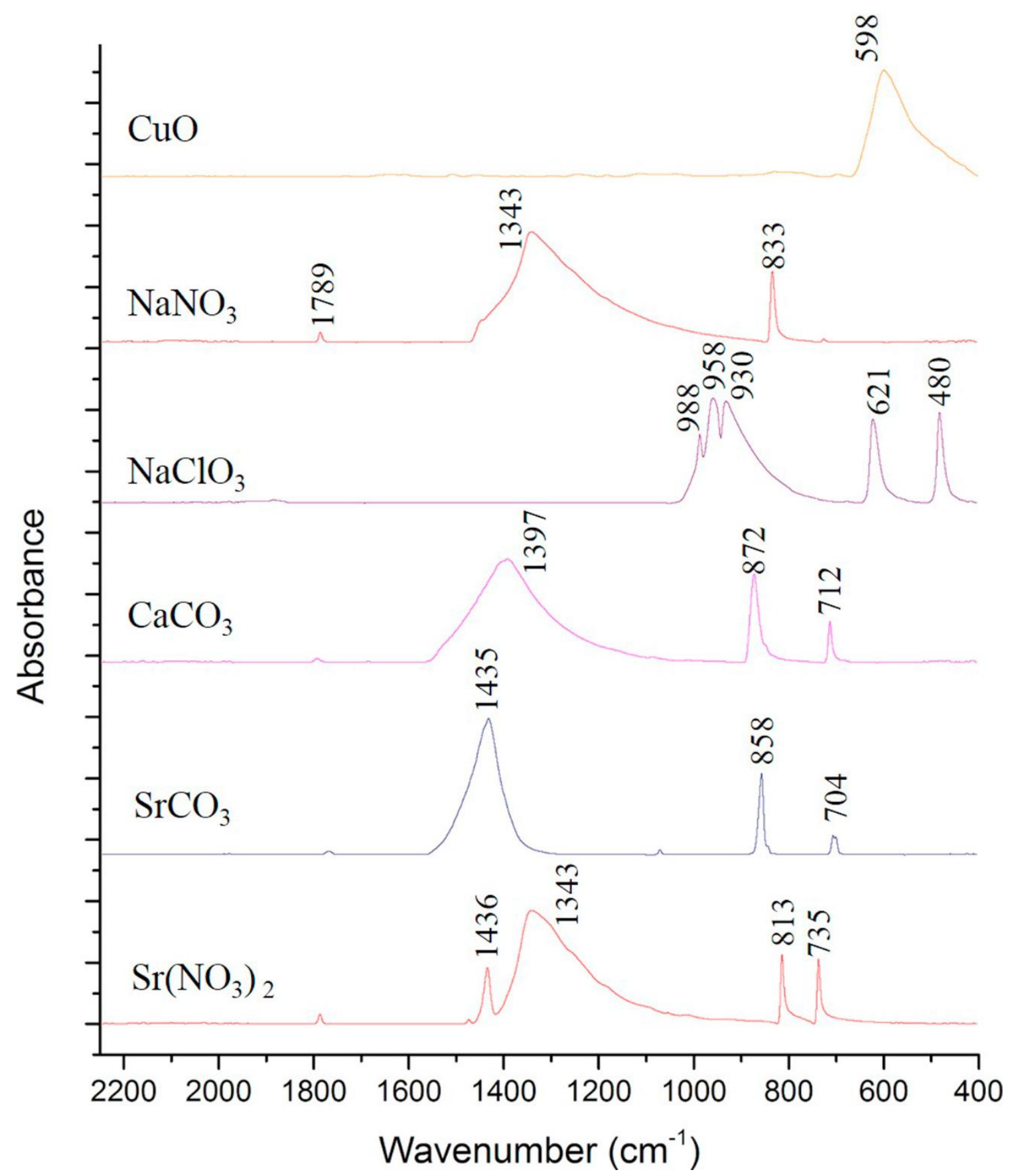

Figure S1 (supplementary material). ATR-FTIR absorption spectra for the standard reagents less frequently used in pyrotechnic mixtures of consumer fireworks. 